TecnoLógicas

ISSN-p 0123-7799

ISSN-e 2256-5337

Vol. 22, No. 45, pp. 21-43

Mayo-agosto de 2019

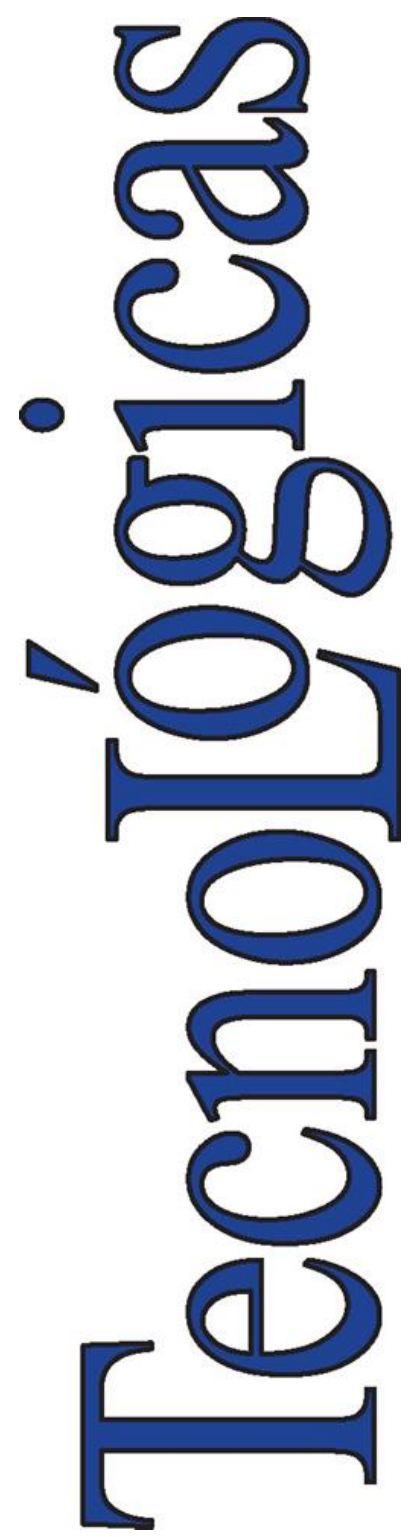

(C) Instituto Tecnológico Metropolitano Este trabajo está licenciado bajo una Licencia Internacional Creative

Commons Atribución (CC BY-NC-SA)

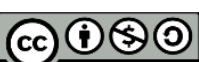

Artículo de Investigación/Research Article

\section{Herramienta informática para notificación comunitaria como insumo para la generación de alertas en seguridad alimentaria y nutricional}

\section{A mobile app to issue warning for food and nutrition security}

\author{
Andrés Vera-Buitrago (D1, y \\ Jorge E. Camargo-Mendoza(D)
}

Recibido: 01 de enero de 2019

Aceptado: 05 de marzo de 2019

\section{Cómo citar / How to cite}

A. Vera-Buitrago, J. E. Camargo-Mendoza, "Herramienta informática para notificación comunitaria como insumo para la generación de alertas en seguridad alimentaria y nutricional," TecnoLógicas, vol. 22, no. 45 , pp. 21-43, 2019. https://doi.org/10.22430/22565337.1174
1 Ingeniero de Sistemas, Departamento de Ingeniería de Sistemas e Industrial, Universidad Nacional de Colombia, Bogotá-Colombia, anverabu@unal.edu.co

2 PhD en Física, Departamento de Física, Universidad Nacional de Colombia, Bogotá-Colombia, jecamargom@unal.edu.co 


\title{
Resumen
}

Este documento presenta la aplicación móvil EVANI, como herramienta informática de apoyo a las comunidades, que les permita en sus territorios la identificación de casos de desnutrición en niños y niñas menores de 60 meses de edad, así como el reporte de las coordenadas del caso y la generación de alertas ante el riesgo de mortalidad derivada de esta causa. Una vez desarrollada la aplicación, se realizó un piloto para validar el funcionamiento de la app y tratar de identificar el posible impacto, obteniendo como resultado, su utilidad en casos de alerta temprana. Lo anterior permitió identificar la viabilidad de EVANI como una herramienta de notificación de alertas nutricionales, las cuales se constituyen en un elemento necesario dentro de los sistemas de alertas tempranas en Seguridad Alimentaria y Nutricional del país, que permita a los diferentes sectores de Gobierno brindar de manera oportuna la atención necesaria para evitar la mortalidad por desnutrición.

\section{Palabras clave}

Desnutrición infantil, alertas tempranas, aplicación móvil, herramienta informática, Seguridad Alimentaria y Nutricional.

\begin{abstract}
This document presents the mobile application EVANI, as a solution to support communities, which allows them in their territories, the identification of cases of malnutrition in children under 60 months of age, as well as the report of the coordinates of the case and the generation of alerts against the risk of mortality derived from this cause. Once the application was developed, a pilot was conducted to validate the operation of the app and try to identify the possible impact, obtaining as a result, its usefulness in cases of early warning. This allowed us to identify the viability of EVANI as a nutritional alert notification tool, which is a necessary element within the early warning systems of food and nutritional security in the country, which allows the different sectors of government to provide in a timely manner, the necessary care to avoid mortality due to malnutrition.
\end{abstract}

\section{Keywords}

Child malnutrition, early warnings, mobile application, computer tool, food and nutritional security. 
Herramienta informática para notificación comunitaria como insumo para la generación de alertas en seguridad alimentaria y nutricional

\section{INTRODUCCIÓN}

La motivación principal de este trabajo es dar un aporte a la sociedad que ayude a salvar vidas, por medio de la informática aplicada. Una oportunidad para esto es encaminarse por la línea temática de la Seguridad Alimentaria y Nutricional, ya que es una realidad que la desigualdad genera muchos casos de hambruna y muertes por temas relacionados a la pobreza [1].

"En 2017, el 7,5 \% de los niños menores de cinco años (50,5 millones) se vieron afectados por la malnutrición aguda, lo que los hace padecer un mayor riesgo de mortalidad" [2].

Por otra parte, según el boletín de las TIC, que entrega el Departamento Administrativo Nacional de EstadísticasDANE, en el año 2017, del total nacional, el 96,4 \% de los hogares tenía por lo menos un teléfono celular; 97,5\% en las cabeceras y $92,2 \%$ en centros poblados y rural disperso [3]. Esto da un reflejo de la posibilidad de comunicación por medio de las redes celulares. Si bien no todos tienen la posibilidad de acceso a internet desde su celular, sí tienen la capacidad de enviar mensajes de texto.

También el DANE entrega indicadores sobre el tipo de dispositivo que utiliza la población cuando accede a internet. El teléfono celular es el dispositivo más utilizado y los centros poblados y rural disperso tienen una participación importante [3], lo que da fuerza al planteamiento de una aplicación móvil como mecanismo de apoyo para mejorar y el acceso a servicios de salud y generación de alertas tempranas [4].

Lo anterior lleva a desarrollar la propuesta de una herramienta informática de tipo aplicación móvil, que captura datos en procesos de tamizaje nutricional de menores de 60 meses, y según los criterios del Ministerio de Salud y Protección Social, y las tablas antropométricas de la Organización Mundial de la Salud, determina si existe una alerta temprana para reducir el riesgo de mortalidad infantil. Esta herramienta funciona con acceso del dispositivo a internet sin embargo está en la posibilidad de generar una alerta por medio de mensaje de texto (SMS) con las coordenadas del caso, cuando en la zona no exista cobertura de datos móviles.

Este sistema, de ser adoptado por alguna entidad pública $u$ organización privada, ayudará a reducir la mortalidad infantil por desnutrición, ya que permite que la población objetivo y en especial en las áreas rurales, acceder a un mecanismo de notificación de casos para recibir la atención oportunamente, y así avanzar en pro de la defensa del derecho a la alimentación y del derecho a la vida.

Como contexto político, en Colombia en el año 2008 el Consejo Nacional de Política Económica Social (CONPES), a cargo del Departamento Nacional de Planeación, emite el documento 113, donde se trata la Política Nacional de Seguridad Alimentaria y Nutricional (SAN). En la construcción de este documento participan el Ministerio de Salud, el Ministerio de Agricultura, el Ministerio de Educación, el Instituto Colombiano de Bienestar Familiar y el Instituto Colombiano de Desarrollo Rural. Este documento plantea la siguiente definición:

"Seguridad alimentaria y nutricional es la disponibilidad suficiente y estable de alimentos, el acceso y el consumo oportuno y permanente de los mismos en cantidad, calidad e inocuidad por parte de todas las personas, bajo condiciones que permitan su adecuada utilización biológica, para llevar una vida saludable y activa" [2].

Posteriormente, y tomando como base las líneas políticas que entrega este documento, el Estado colombiano genera el decreto 2055 de 2009, donde crea la Comisión Intersectorial de Seguridad Alimentaria y Nutricional - CISAN, la cual fue modificada mediante el decreto 1115 de 2014 , como resultado de la expedición de la 
Herramienta informática para notificación comunitaria como insumo para la generación de alertas en seguridad alimentaria y nutricional

Ley 1355 de 2009. Esta instancia se encuentra conformada por las siguientes entidades: Ministerio de Agricultura y Desarrollo Rural; Ministerio de Salud y Protección Social; Ministerio de Comercio, Industria y Turismo; Ministerio de Educación Nacional; Ministerio de Ambiente y Desarrollo Sostenible; Ministerio de Vivienda Ciudad y Territorio; Departamento Nacional de Planeación; Instituto Colombiano de Bienestar Familiar -ICBF; Departamento Administrativo para la Prosperidad Social -DPS; Instituto Colombiano de Desarrollo Rural -INCODER; y un miembro de la Asociación Colombiana de Facultades de Nutrición, designado por su Junta Directiva [3].

Esta comisión tiene dentro de sus principales funciones: elaborar el plan nacional de SAN, tomando las líneas políticas establecidas en el documento CONPES 113; articular el plan con las políticas de desarrollo del país; coordinar el proceso de inclusión de nuevos programas y proyectos; fortalecer capacidades institucionales en SAN La eSalud: territorial en sector público y privado; proponer mecanismos de seguimiento y evaluación; promover intercambio de experiencias; promover la creación del observatorio de seguridad alimentaria y nutricional; crear instancias para el seguimiento y control por parte de comunidades implicadas [3].

Posteriormente, en el año 2012, se aprueba el plan nacional de SAN (20122019), el cual plantea la necesidad de un sistema de alertas tempranas en efectos climáticos y de disponibilidad estable del grupo de alimentos prioritarios.

De igual forma, este documento, realiza un contexto de la situación del país en tema de SAN y explica los ejes que la componen: Disponibilidad, Acceso, Inocuidad, Consumo y Aprovechamiento Biológico; este último muestra la utilización de nutrientes de los alimentos consumidos en la población [4].
Así, consecuentemente, el 13 de marzo de 2013 el Gobierno colombiano lanza el Observatorio de Seguridad Alimentaria y Nutricional-OSAN, cumpliendo con lo dispuesto en el decreto de creación de la CISAN. Esta instancia tiene como objetivo la creación de "un sistema integrado de instituciones, actores, políticas, procesos, tecnologías, recursos y responsables de la SAN, que integre, produzca y facilite el análisis de información y gestión del conocimiento, con el objetivo de facilitar el proceso de diseño, implementación, seguimiento y evaluación de la SAN, de su política (PSAN), y de las acciones que buscan garantizarla" [5].

Fruto de estas asignaciones el OSAN genera un documento en el año 2013, llamado "Documento metodológico para la construcción del subsistema de alertas en la seguridad alimentaria y nutricional", en donde se plantea la estrategia a seguir para construir un sistema de alertas que dé cuenta de los ejes temáticos de la seguridad alimentaria y nutricional, teniendo presente la acción de cada uno de los actores implicados, tanto del sector público, privado y comunidades [5].

El siguiente año (2014), el OSAN, presentó el primer informe del subsistema de alertas en SAN, a partir de indicadores de sistemas de información existentes en Colombia, no necesariamente reportados de manera periódica; este se planteó como un ejercicio de implementación de su primera fase de operación, donde posteriormente se espera poder generar información a través de otras fuentes, destacando procesos en los cuales se pueda vincular a la sociedad civil, mediante una estrategia de notificación comunitaria [6]; no obstante, dentro de la revisión realizada, posterior a este informe, no se han vuelto a generar más reportes del subsistema. 
Herramienta informática para notificación comunitaria como insumo para la generación de alertas en seguridad alimentaria y nutricional

\section{ESTADO DEL ARTE}

Actualmente, en Colombia no existe un sistema de vigilancia alimentaria $y$ nutricional, que permita de manera periódica conocer la situación del estado nutricional de la población, desagregado a nivel departamental, municipal $y$ en poblaciones vulnerables, como elemento central de alertas, para la implementación de acciones oportunas que permitan avanzar en la garantía del derecho a la alimentación en el país [7].

Con el fin de mejorar este proceso, desde el año 2014, el OSAN avanzó en la primera fase de implementación de un subsistema de alertas tempranas en seguridad alimentaria y nutricional, el cual fue descrito anteriormente.

No obstante, aún no se han generado acciones para incorporar un componente de notificación comunitaria, especialmente de situaciones que puedan poner en riesgo la vida de las personas, que implica una obligación de cumplimento inmediato por parte de los Estados, según lo establecido para la garantía del derecho humano a la alimentación y nutrición adecuadas [8].

Una de las situaciones de riesgo, corresponde a las deficiencias nutricionales en la población menor de cinco años, las cuales pueden llevar fácilmente a la mortalidad temprana, como es el caso de la desnutrición aguda [9]; por ello, es el interés particular de este trabajo desarrollar una herramienta que permita a la sociedad civil realizar seguimiento del estado nutricional a nivel comunitario, con el fin de generar, de manera sencilla, alertas oportunas que permitan a las instituciones desplegar las acciones necesarias para dar respuesta a esta situación.

Basado en lo anterior, se presentan a continuación las experiencias identificadas tanto a nivel nacional como internacional, en el uso de herramientas informáticas para la notificación de alertas nutricionales de tipo comunitario, en el marco de la Seguridad Alimentaria y Nutricional, para la identificación tanto de los avances, como de las debilidades en torno a las mismas.

Como punto de partida, International Telecommunication Union, publica "Soluciones de eSalud móvil para los países en desarrollo", donde se plantean preguntas clave sobre el uso de varias tecnologías, entre estas SMS y GPS, como apoyo a las actividades en el área de la salud [10]. Estas tecnologías son clave dentro de la propuesta expuesta unos párrafos más adelante.

La Organización de las Naciones Unidas para la Agricultura y la Alimentación - FAO, en el 2001, propuso la creación del Sistema de Información para la Seguridad Alimentaria y Alerta Temprana - SISAAT, el cual incorpora cuatro componentes, 1) el seguimiento a la producción agrícola, 2) el sistema de información sobre los mercados, 3) seguimiento a grupos expuestos a riesgos donde se miran aspectos como pobreza, cantidad y calidad de los alimentos consumidos, empleo, entre otros estabilidad, acceso, y 4) el seguimiento alimentario y nutricional, en el cual se incorpora la información relacionada con estado nutricional y para este caso, dentro de los instrumentos resaltados, se encuentran los sistemas de seguimiento comunitario, los cuales dependen de proyectos o programas de desarrollo que estén en marcha, por lo que no se constituyen en un mecanismo permanente $[11$, p. 53] [12].

Esta propuesta plantea la necesidad de tomar datos nutricionales cada dos meses o menos. Para ello, se recolectan y organizan los datos, mediante el uso de técnicas y software diseñados para encuestas y el manejo de datos. Posteriormente, se analizan para poderlos divulgar a los tomadores de decisiones y así generar medidas para resolver el problema [11]; sin embargo, este proceso no facilita de manera rápida el reporte de la alerta, pues 
Herramienta informática para notificación comunitaria como insumo para la generación de alertas en seguridad alimentaria y nutricional

se debe surtir el proceso de digitación, depuración de las bases de datos, análisis y elaboración de informes para su posterior notificación.

Posteriormente, Acción Contra el Hambre-ACH—, en el 2013, construyó en Guatemala una guía para la implementación de sitios centinela en ese país, la cual contempla la generación de alertas a partir de una propuesta de trabajo comunitario, en donde la desnutrición aguda fue uno de los indicadores seleccionado para la notificación, no obstante la toma de datos estaba bajo la responsabilidad de los promotores de salud y los agentes comunitarios debían buscar el dato, situación que no permite el reporte permanente y oportuno de casos de desnutrición [13].

De igual manera, el Programa Mundial de Alimentos -PMA—, en su informe del año 2017, reconoce la importancia de los Sistemas de Alertas Tempranas - SATpara salvar vidas, y por ello avanzó en la consolidación de una propuesta de SAT para Centroamérica (Guatemala, Honduras, El Salvador y Nicaragua), estructurada en siete fases orientadas a la descripción e identificación de las necesidades de los países en cuanto a información y clasificación de las alertas, así como los protocolos de respuesta y rol de los actores [14], sin embargo, la información nutricional identificada corresponde a los sistemas de información con periodos de reporte anuales, los cuales no permiten el monitoreo permanente $\mathrm{y}$ generación de alertas tempranas, particularmente para estos indicadores, ni plantean claramente un proceso de participación comunitaria para su funcionamiento.

En Nicaragua, se han tomado acciones para dar oportunidad a las poblaciones rurales de tener un enlace comunicativo por medio de redes WiMAX/Wifi y así, con el uso de estas tecnologías, informar eventos de salud prioritarios tipo materno- infantil y ser atendidos por el personal dispuesto en los centros de salud cercanos [15].

Existen, además, otros referentes como el Sistema de Información y Cartografía sobre la Inseguridad Alimentaria y Vulnerabilidad, el Sistema Mundial de Información y Alerta sobre la Alimentación y la Agricultura (SMIA), el Sistema de Alerta Temprana para Centroamérica (SATCA) y el Sistema Mesoamericano de Alerta Temprana para la Seguridad Alimentaria (MFEWS), entre otros, los cuales reportan información para prevenir y/o atender crisis alimentarias; de estas experiencias se destaca el uso de sistemas de información geográfica, que permiten identificar las zonas con mayor riesgo; sin embargo pese a su existencia, se siguen evidenciando situaciones relacionadas con hambre y falta de respuestas oportunas, y no se cuenta con uso de herramientas informáticas que generen una alerta inmediata a las instituciones encargadas de la atención.

En Colombia existen diferentes iniciativas que incorporan sistemas de información en SAN, en donde se destaca el Sistema de Vigilancia en Salud Pública (SIVIGILA) y el Sistema de Vigilancia Alimentario y Nutricional (SISVAN), los cuales cuentan con indicadores del estado nutricional, aunque la periodicidad y forma de toma de los datos no permiten generar alertas tempranas [16].

Se han desarrollado otras herramientas de alertas tempranas en SAN, orientadas principalmente hacia los riesgos climáticos y su afectación en la producción de alimentos, los cuales han sido trabajados por el Ministerio de Agricultura y Desarrollo Rural (MADR), pero estos no incorporan el componente nutricional.

Adicionalmente, la Defensoría del Pueblo cuenta con el Sistema de Alertas Tempranas, que se define como el instrumento con el que esta institución acopia, verifica y analiza, de manera técnica, información relacionada con 
situaciones de vulnerabilidad y riesgo de la población civil como consecuencia del conflicto armado, y advierte a las autoridades concernidas con deber de protección para que se coordine y brinde una atención oportuna e integral a las comunidades afectadas. El Ministerio de Salud y Protección Social es una de las entidades que recibe la información reportada por el sistema para brindar respuesta a las mismas [5].

En relación con aplicaciones móviles en el tema alimentario para la generación de alertas, solamente se encontró la experiencia de la Red de Información y Comunicación Estratégica del Sector Agropecuario AGRONET, una iniciativa del MADR articulada con el plan nacional vive digital Colombia, la cual hace uso de tecnologías como CELUAGRONET, la cual, a través de mensajes de texto vía celular, permite informar a los productores agropecuarios para la toma de decisiones [5].

Finalmente, con la revisión realizada, se encuentra que se han presentado avances en la generación de sistemas de alertas tempranas en seguridad alimentaria y nutricional, estos son en su mayoría institucionales, sin desconocer los esfuerzos para el avance hacia la incorporación de un componente de participación comunitaria.

A nivel académico, se encuentra una tesis de grado de Ingeniería de Sistemas, de la Universidad Nacional José María Arguedas de Perú, donde se presenta una aplicación móvil que realiza evaluación nutricional infantil para apoyar la verificación de datos de un programa nacional en este país, sin embargo, este no actúa como sistema de alertas tempranas y funciona bajo el acceso de internet [17].

Pese a lo anterior, no se encontraron experiencias de aplicaciones para la generación de alertas nutricionales, haciendo uso de herramientas informáticas, lo cual se constituye en una oportunidad para incorporar el uso de las tecnologías de la información y las comunicaciones, como herramientas de innovación social, que permitan involucrar a la sociedad civil como un sujeto activo, partícipe de la identificación de sus problemas, así como de las soluciones a los mismos, mediante el reporte de alertas nutricionales que faciliten a las instituciones, la respuesta inmediata a situaciones urgentes, localizando a las personas afectadas rápidamente [18].

Lo anterior, como una apuesta que permita el cierre de brechas sociales en Colombia, donde no haya más niños y niñas que mueran de hambre, y permita de esta forma avanzar en la garantía del derecho a la alimentación y nutrición adecuadas para toda la población colombiana.

\section{CONTEXTO DEL TRABAJO}

\subsection{Estado inicial}

Para tener datos de referencia, y según la Encuesta Nacional de la Situación Nutricional en Colombia -ENSIN_, 2015, el país tiene un $54,2 \%$ de la población en algún estado de inseguridad alimentaria, distribuido más específicamente de la siguiente manera; $31,9 \%$ en inseguridad leve, $13,8 \%$ en inseguridad moderada y 8,5 $\%$ en inseguridad severa [19].

También, según los datos del Instituto Nacional de Salud a corte de octubre de 2018, se presentan en la Tabla 1, los valores porcentuales de distribución de muertes en menores de 5 años para los eventos priorizados.

Tabla 1. Muertes por DNT, menores de 60 meses año 2018. Fuente: [14].

\begin{tabular}{ccccc}
\cline { 2 - 5 } & \multicolumn{2}{c}{ DNT2017 } & \multicolumn{3}{c}{ DNT2018 } \\
\hline Genero & Casos & $\%$ & Casos & $\%$ \\
Femenino & 79 & 48 & 107 & 53 \\
Masculino & 84 & 52 & 96 & 47 \\
\hline
\end{tabular}


Herramienta informática para notificación comunitaria como insumo para la generación de alertas en seguridad alimentaria y nutricional

Tomando la información anterior y volviendo al contexto político, en enero de 2015 se establecen los 17 objetivos de desarrollo sostenible - ODS - liderado por el PNUD, el cual se convierte en un marco de compromiso a nivel internacional, el cual fue firmado por Colombia y generó la elaboración de una estrategia nacional de implementación para su cumplimiento, consignada en el CONPES 3918 de 2018 [20].

Particularmente, para este trabajo, se destacan dos de estos objetivos: Objetivo 2, "Poner fin al hambre, lograr la seguridad alimentaria y la mejora de la nutrición y promover la agricultura sostenible; y Objetivo 3, "Garantizar una vida sana y promover el bienestar para todos en todas las edades", con las siguientes metas trazadas para el año 2030. De esto objetivos se resaltan los siguientes párrafos [20]:

-Objetivo 2: Para 2030, poner fin a todas las formas de malnutrición, incluso logrando, a más tardar en 2025, las metas convenidas internacionalmente sobre el retraso del crecimiento y la emaciación de los niños menores de 5 años y abordar las necesidades de nutrición de las adolescentes, las mujeres embarazadas y lactantes y las personas de edad.

-Objetivo 3: Para 2030, poner fin a las muertes evitables de recién nacidos y de niños menores de 5 años, logrando que todos los países intenten reducir la mortalidad neonatal al menos hasta 12 por cada 1.000 nacidos vivos, y la mortalidad de niños menores de 5 años al menos hasta 25 por cada 1.000 nacidos vivos.

Pueden existir diferentes factores que alteren cada componente de la SAN, y parten de causas primarias que pueden evolucionar a escenarios más complejos donde aparecen casos de mortalidad.
Por otro lado, el Observatorio de Soberanía y Seguridad Alimentaria y Nutricional -OBSAN_ de la Universidad Nacional de Colombia, ha trabajado en la "Escuela de líderes gestores y notificadores en SAN", un programa donde se capacitan líderes de comunidades para poder realizar notificaciones a los entes territoriales encargados sobre la situación de SAN en sus territorios. Estas personas capacitadas son un engranaje importante en la detección de casos y reporte oportuno, que debería ser una estrategia incorporada dentro de los procesos de desarrollo de capacidades en los territorios, y a su vez permita avanzar en la generación de mecanismos para la exigibilidad de los derechos en las diferentes poblaciones del país [21].

Claramente, el subsistema de alertas tempranas debe tener varios actores comprometidos y cada uno cumpliendo un rol específico dentro del esquema, que permita mantener una sinergia entre las acciones para reducir los indicadores que dan cuenta al estado de SAN, y apoyados en diferentes recursos humanos $y$ múltiples herramientas de alcance técnico y tecnológico, donde haya un trabajo colaborativo entre las entidades de Gobierno y la sociedad civil.

Como fue presentado en el estado del arte, si bien existen sistemas de alertas en SAN, estos no han hecho uso de aplicaciones móviles, sino que se han planteado desde diferentes metodologías de recolección de información; no obstante, para temas como la gestión del riesgo relacionados con desastres o el clima, sí se han venido implementando algunas herramientas móviles, las cuales han permitido vincular a las poblaciones en su notificación.

Las aplicaciones móviles se han convertido en una herramienta que "permite resolver problemas de la sociedad, debido a su facilidad de movilidad $y$ ubicuidad [22]". Además, es una alternativa que ya se ha reconocido como 
útil en la práctica médica y en salud pública por la Organización Mundial de la Salud, que ha implementado estrategias apoyados en dispositivos móviles y otros artefactos electrónicos [23], que hacen viable y necesaria su implementación en Colombia para el tema de SAN, particularmente en lo nutricional, que sigue cobrando vidas de niñas y niños, especialmente en zonas lejanas del país.

\section{2 ¿Por qué desarrollar una app?}

De lo anterior, se puede evidenciar la necesidad de que en Colombia se implemente el subsistema de alertas tempranas. Gran parte de la labor está en el territorio, que es donde se presentan los casos de vulnerabilidad para cada uno de los ejes temáticos de la SAN, y se deben ofrecer herramientas para poder realizar los reconocimientos y difusiones de las eventualidades y el monitoreo y control de las acciones por parte del estado. Estas acciones deben ser trabajadas de manera conjunta con las personas que habitan en los territorios, lo que implica un proceso del Estado con un rol de promotor de nuevas formas de organización de la vida social, que permita el empoderamiento y formación de sujetos activos que incidan en la gestión de lo social [24].

Como lo indica el OSAN, y con el fin de medir, controlar, preparar y responder ante una emergencia de SAN, se debe implementar una metodología de alertas tempranas de este tipo en el país; aunque existen sistemas que manejan temas afines a los componentes de la SAN, estos no ofrecen alertas en periodos cortos, ni las herramientas suficientes para realizar $\mathrm{y}$ supervisar todo el proceso que implica una alerta temprana [5].

En el territorio existen líderes comunitarios que están en capacidad de interactuar en los eventos relacionados con las alertas en SAN, por lo tanto, ¿cómo apoyar el proceso de reporte de alertas tempranas a los gestores locales en SAN, para que puedan ser validadas, divulgadas, obtener seguimiento y control por parte de las entidades responsables de atender la alerta?

En este punto surge el planteamiento de diseñar una herramienta informática de tipo aplicación móvil, que permita la notificación comunitaria de posibles casos de desnutrición infantil por parte de los gestores en SAN en territorio, y así aumentar la posibilidad de que puedan ser validadas y atendidas oportunamente por parte de los entes estatales encargados (Fig. 1), generando procesos de innovación social.

\subsection{Alcance}

El sistema de notificación comunitaria de alertas por los gestores en SAN está pensado en diferentes temáticas $y$ variables (escalable), sin embargo, se inicia con la propuesta de un prototipo que pueda ser usado para obtener la pertinencia, funcionalidad y tal vez una aproximación del impacto que puede tener. Por lo anterior, se plantea una fase inicial tomando el indicador de desnutrición crónica y aguda en niños y niñas menores de 5 años. Por ser uno de los temas más críticos, al tratarse de niñez y posibles casos que pueden llegar a la mortalidad, se planteó este como punto inicial para desarrollar la aplicación móvil, en adelante referenciada como EVANI.

El sistema es una herramienta para el ingreso de datos de tamizaje realizado por los gestores, el cual calcula el estado de los niños y niñas menores de 60 meses de edad, teniendo presente las tablas de referencia de patrones de crecimiento de la OMS y citadas en la resolución 2465 del de 2016 del Ministerio de la Protección Social [25]. Una vez realizada la evaluación, se hace un reporte a una base datos centralizada y según la situación del menor generará mensaje de alerta a los usuarios 


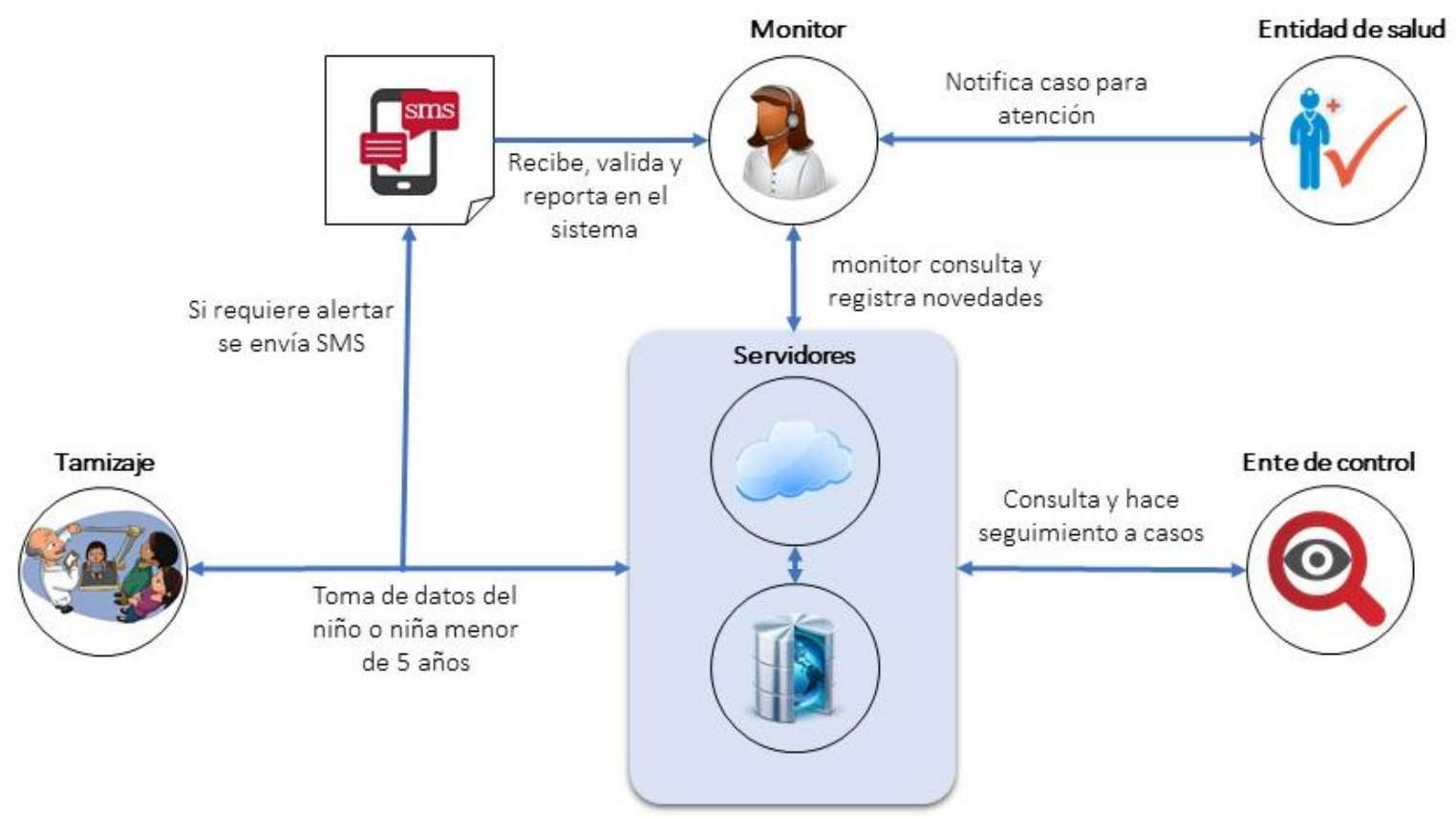

Fig. 1. Esquema operacional de EVANI. Fuente: autor.

asignados como rol responsable de la visualización del caso. Adicionalmente, entidades de control pueden tener usuarios con el rol que permite realizar un seguimiento de las atenciones para garantizar los derechos fundamentales de las personas que requieren atención. La herramienta facilitará los temas de reporte, difusión y control por medio de seguimiento histórico de casos, pero jamás garantizará la respuesta inmediata ni la toma de acciones; estas tareas son de las entidades asignadas como responsables.

\section{DESARROLLO}

\subsection{Requisitos}

Para este propósito, se trabajó con algunos artefactos de UML (Unified Modeling Language). Se trabaja con una muestra de datos base que se incorpora al sistema para validar el funcionamiento. Para estos casos registrados se generan reportes estadísticos generales, mas no reportes detallados, con el fin de no vulnerar ningún derecho a las personas que tenga que ver con su condición de salud y/o información personal. Los artefactos que se trabajaron son: especificación de requerimientos, diagramas de caso de uso para la etapa de elaboración y documento de arquitectura, que trabaja con la vista de clases con el modelo Entidad-Relación y la vista de implementación con los diagramas de secuencia, estados y colaboración, vista conceptual con el diagrama de clases, vista física con el esquema de hardware. Posterior al desarrollo, se implementa un piloto con 22 niños y niñas menores de 60 meses de edad.

Los requerimientos del sistema, como es habitual en desarrollo de sistemas de información, se separaron en funcionales y no funcionales, de esta clasificación nacen ocho fichas de casos de uso que obedecen a las siguientes condiciones: es una aplicación móvil funcional bajo el sistema operativo Android 5.0 o superior (Fig. 2), con acceso a internet o mínimo con acceso a red telefónica para envío de SMS.

Captura la información de cada persona que interactúe en la aplicación. Si es el registro de una madre o un padre, hace el 
registro y continúa con el ingreso de los datos del niño o la niña. Posteriormente, obtiene la geolocalización usando el GPS del dispositivo (Fig. 3) y solicitar enseguida los datos del tamizaje, como peso, talla, perímetro braquial y cefálico, también la opción de indicar presencia de edema en alguna parte del cuerpo y agregar un texto con observaciones.

Si al terminar el proceso de evaluación se identifica que cumple los criterios para envío de alerta, se enviará alerta por medio de SMS (Fig. 4) con la ubicación geográfica del caso para identificar por medio del servicio de Google Maps. Este caso es recibido por el agente encargado para validación de casos; este, a su vez, debe avisar al ente idóneo para la atención de dicho caso.

EVANI también genera el reporte estadístico nacional, por departamento o municipio, de los casos reportados y el estado en el que se encuentren a la fecha de la consulta (Fig. 5). También puede consultar la gráfica de las curvas de los $\mathrm{Z}$ Score, de la OMS.

Existe la opción de validación de los casos, que podrá asignar un nuevo estado según la evolución que este tenga.

El sistema habilita funcionalidades según el rol al que pertenece cada usuario, estos roles son: Administrador. que tiene el permiso a todas las acciones de la app; Tamizaje, que tiene habilitadas las opciones de registro de datos de personas y capturas antropométricas; Monitor, que permite ver algunos reportes y registrar novedades sobre los casos; finalmente, Organización, que tiene solo acceso a consulta para seguimiento de casos.
El sistema requiere que cada usuario al que se le otorgue acceso pase por un proceso de validación de perfil y que pertenezca a una organización comunitaria, entidad estatal o internacional que vele por el bienestar de los niños y niñas. Por otra parte, al estar dirigido para un trabajo en territorio tiene un módulo de sincronización manual para retroalimentar los datos al servidor, cuando el dispositivo móvil hubiese tenido que hacer captura de datos desconectado de internet.

\subsection{Recursos}

Para el desarrollo y puesta en marchar del piloto de EVANI se necesitaron recursos humanos y material/lógico. El recurso humano contó con el apoyo de nutricionistas, arquitecto de software, expertos en políticas públicas en SAN, diseñadores gráficos, desarrollador de software y de bases de datos y el apoyo de enfermeras y maestras en las jornadas de tamizaje del piloto.

El recurso material/lógico fue: servicio de hosting que atienda 2 capas una de base de datos y otra de presentación, con condiciones de servicio que garanticen la seguridad de la información y la integridad del sistema y tiempos de respuesta adecuados a cada requerimiento técnico. Dispositivos móviles con GPS y acceso a red móvil para ejecución de pruebas. Equipos de cómputo para el desarrollo del sistema. Se requiere capacitación para las personas que participen en las pruebas de la aplicación, o que tengan el conocimiento sobre tamizajes nutricionales en primera infancia. 
Herramienta informática para notificación comunitaria como insumo para la generación de alertas en seguridad alimentaria y nutricional

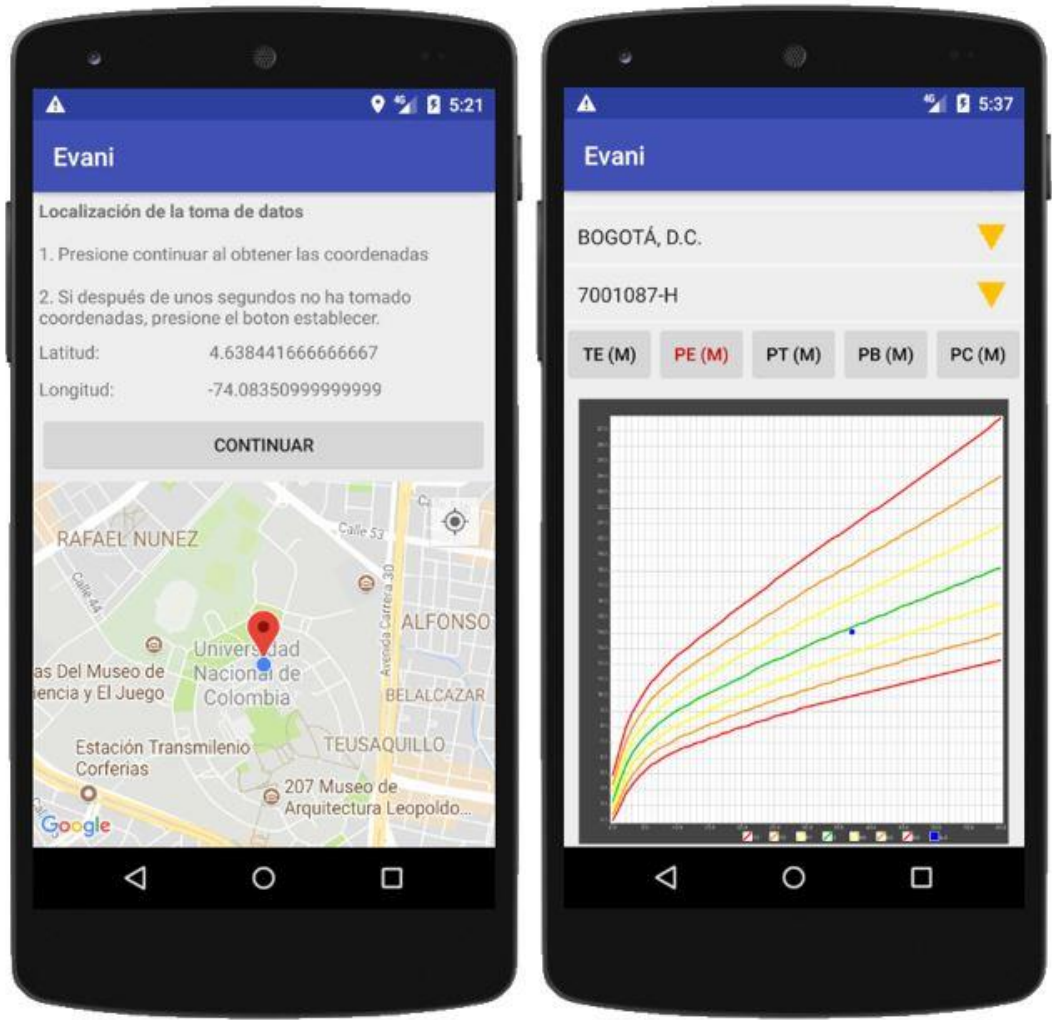

Fig. 2. Capturas de pantalla de EVANI, pantalla de log-in y menú principal. Fuente: autor.

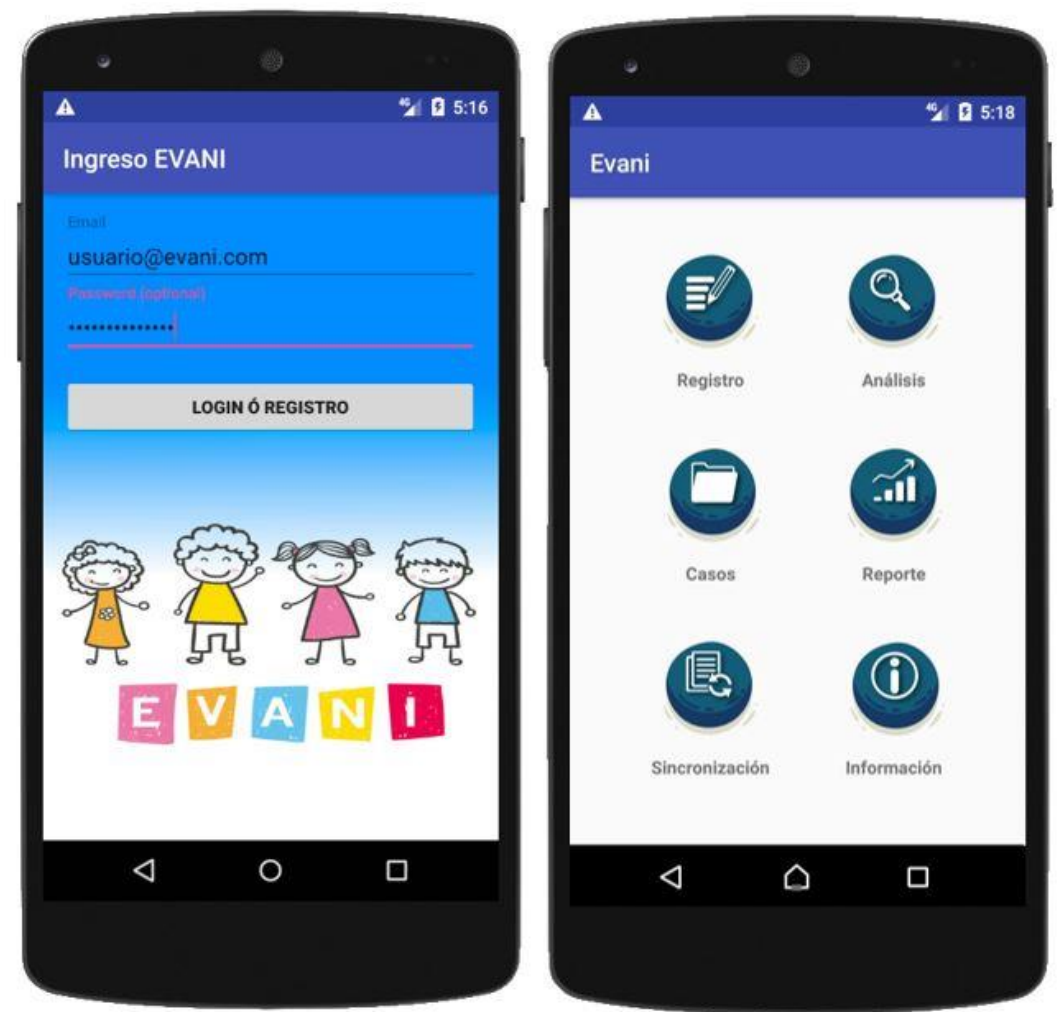

Fig. 3. Capturas de pantalla de EVANI, pantalla de geolocalización y graficas de tablas OMS. Fuente: autor. 
Herramienta informática para notificación comunitaria como insumo para la generación de alertas en seguridad alimentaria y nutricional

\begin{tabular}{lll} 
Alerta & Indicador & Resultado \\
\hline 0 & Talla para la edad & Talla baja para la edad \\
0 & Peso para la edad & Desnutrición global \\
\hline 1 & Peso para la talla & Riesgo de desnutricion aguda \\
\hline 0 & Perímetro cefálico & Factor de riesgo para el neurodesarrollo
\end{tabular}

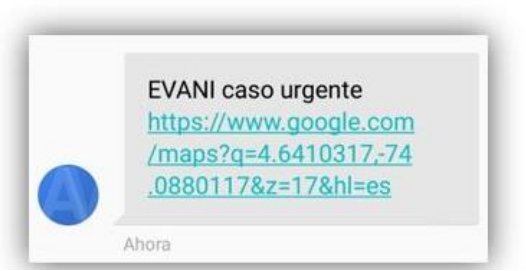

Fig. 4. Capturas de pantalla de EVANI, envío de alerta por SMS. Fuente: autor.

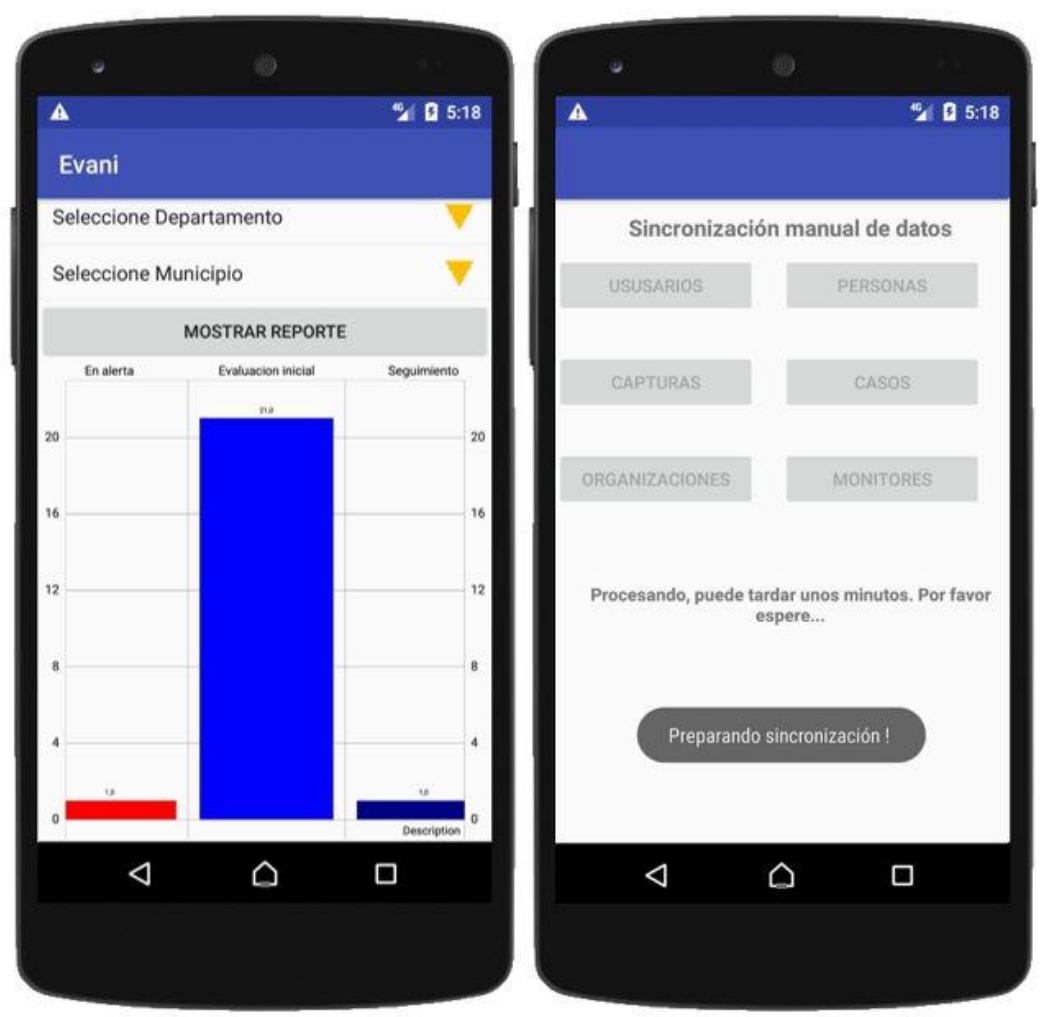

Fig. 5. Capturas de pantalla de EVANI, reporte consolidado y pantalla de sincronización. Fuente: autor.

\subsection{Actividades realizadas}

Se realizaron varias actividades en aproximadamente dos años de trabajo, partiendo de la revisión documental y Contexto legal en temas de nutrición y SAN para obtener un contexto sólido que permitió acentuar más la propuesta, seguido de un análisis de los requerimientos para identificar los perfiles que apoyarían el desarrollo y la implementación. Se realizaron pruebas internas con información histórica obtenida de algunas instituciones que 
Herramienta informática para notificación comunitaria como insumo para la generación de alertas en seguridad alimentaria y nutricional

conocen del tema, y finalmente el desarrollo del piloto en dos instituciones educativas en la ciudad de Bogotá.

Técnicamente se realizaron las siguientes actividades en el desarrollo de la aplicación.

-Requerimientos

Funcionales

No funcionales

-Etapa de diseño

Casos de uso del sistema

Diagrama de entidad - relación - E/R

Diagrama de secuencia

Diagrama de estados

Diagrama de colaboración

Diagrama de componentes

Diagrama de despliegue

Mapa de comportamiento a nivel de hardware

-Etapa de construcción

Tecnologías utilizadas en la capa de base de datos

Tecnologías utilizadas en la capa de aplicación

Tecnologías utilizadas en la capa de presentación

A nivel de diseño, Fig. 6, se esquematizan las capas de despliegue del sistema, donde se tiene como pilar la base de datos retroalimentada por medio de servicios JS con peticiones provenientes de internet, cuando se realiza una actividad de registro o sincronización de datos desde el dispositivo móvil.

\subsection{Tecnologías utilizadas}

EVANI está planteado en su primera versión para funcionar en dispositivos móviles con sistema operativo Android 5.0 o superior, utilizando los servicios de ubicación de Google. Para esto se utiliza el entorno de desarrollo de Android Studio y el lenguaje de programación Java.
Dentro de las librerías necesarias para completar las funcionalidades de la aplicación se incorporan las siguientes:

Librerías nativas de Android que se usan en la creación de componentes básicos de la aplicación móvil, como el soporte para el diseño de la interfaz gráfica, la posibilidad de posicionar elementos de manera flexible y optimizar la navegabilidad HTML. Se pueden definir como las librerías base:

-appcompat-v7:27.1.1:

-constraint-layout:1.1.0:

-design:27.1.1

-support-vector-drawable:27.1.1

-volley:1.0.0

Librerías para la incorporación de gráficos de análisis y de reporte estadístico de casos. Se utilizó MPAndroidChart [26], que es un software gratuito para montar gráficas en Android y la librería java AndroidPlot [27], que también es usada para graficar datos:

-com.github.PhilJay:MPAndroidChart:

v2.2.4

-androidplot-core-0.6.1.jar

Librerías para el uso de los servicios de ubicación de Google, estas librerías requieren un registro dentro de los servicios de Google para poder ser utilizados, tienen una cuota limite gratuita de búsquedas de ubicación por día y están atados a un código ID que identifique la app en donde se usaran:

-com.google.android.gms:play-servicesmaps:9.4.0 -com.google.android.gms:play-serviceslocation:9.4.0

Librerías para la aplicación del consumo de servicio API REST del servidor web de aplicaciones. Se utilizan las librerías de apache y okhttp para el manejo cliente del protocolo http y la librería Gson 
Herramienta informática para notificación comunitaria como insumo para la generación de alertas en seguridad alimentaria y nutricional

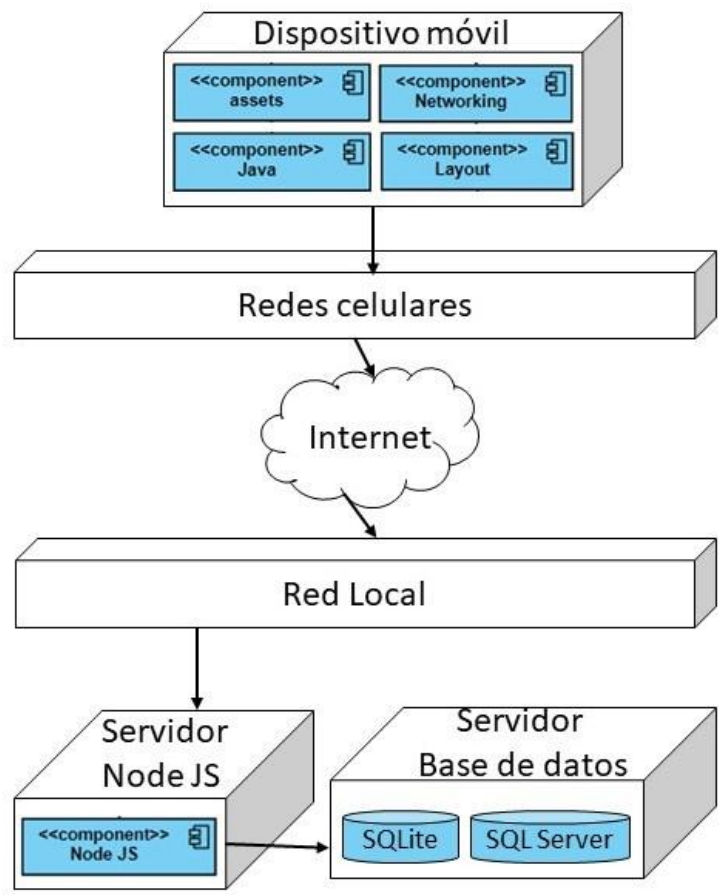

Fig. 6. Despliegue para EVANI. Fuente: autor.

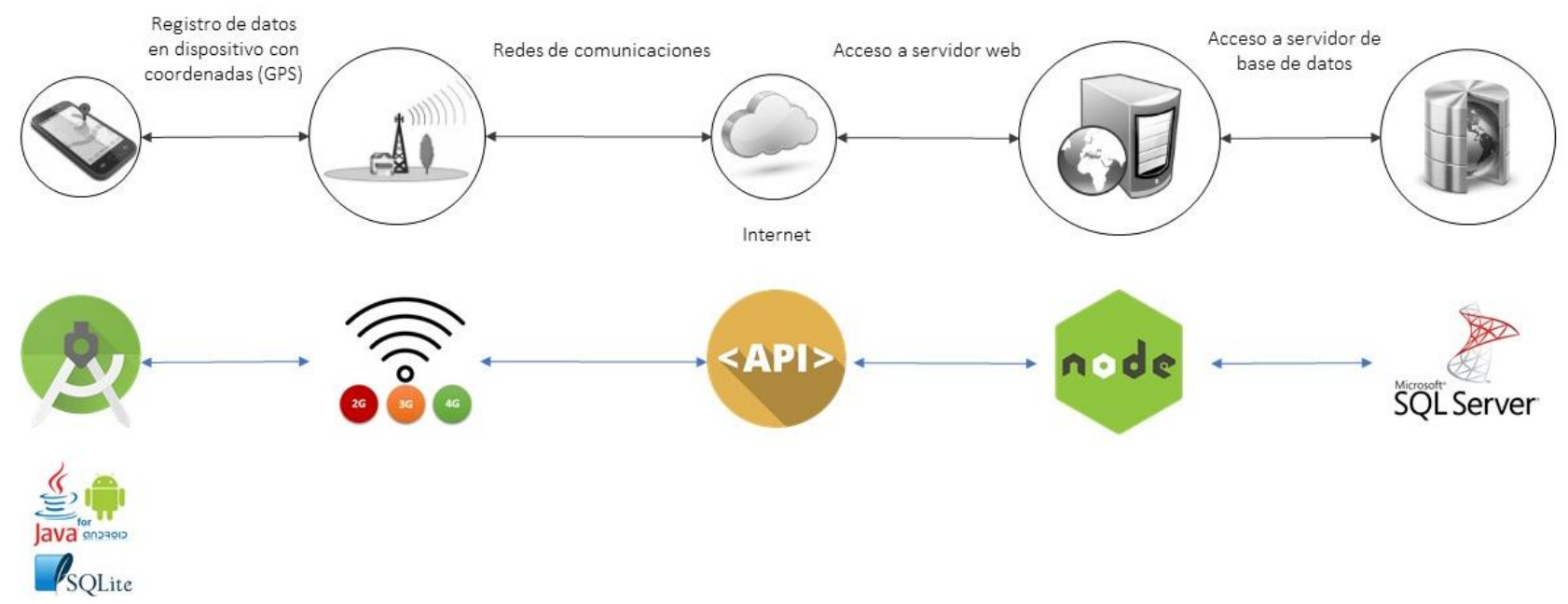

Fig. 7. Tecnologías utilizadas para EVANI. Fuente: autor.

para la gestión de los comandos de solicitud y respuesta al servidor NodeJs:

-org.apache.httpcomponents:httpclientandroid:4.3.5.1

-com.squareup.okhttp3:okhttp:3.4.1

-gson-2.3.1.jar

-com.idescout.sql:sqlscout-server-noop:4.1
Para poder realizar una interacción entre la aplicación y la base de datos se utiliza el componente NodeJs y se dispone en un servidor de tipo API WEB para recibir y atender las peticiones de los dispositivos móviles desde internet.

NodeJs es un entorno de ejecución con base en JavaScript, de licencia libre y parte de los proyectos de Linux 
Herramienta informática para notificación comunitaria como insumo para la generación de alertas en seguridad alimentaria y nutricional

Foundation. Este componente reduce los tiempos de ocupación, ya que es asíncrono y atiende solo cada vez que es invocado. También utiliza diferentes tipos de librerías o paquetes de contenidos que se pueden ir incorporando según las necesidades, para este caso se incorporan las librerías mssql y http. Al ser una aplicación orientada a web y que es de fácil despliegue, es una opción óptima para la implementación de EVANI [28].

Para poder disponer la aplicación servidor NodeJs de EVANI, se utiliza el componente Git que permite iniciar un servidor web application, de manera personalizada y eliminando la necesidad de un servidor web convencional, como IIS para el caso Windows. Esto también genera un procesamiento más liviano $\mathrm{y}$ una codificación más simple gracias a los empaquetados de las dos herramientas utilizadas, ya mencionadas [29].

Finalmente, para la gestión de los datos, se incorpora el servidor de base de datos SQL Server 2014 standar edition. Esta herramienta es robusta y tiene muy buenos tiempos de respuesta, adicionalmente la versión utilizada no genera costos de licenciamiento y permite el desarrollo de la aplicación y una primera fase inicial. Para poder mantener los datos durante los procesos de registro y facilitar una evaluación de alerta en los espacios donde no hay cobertura de internet se utiliza SQLite.

En el diagrama que se muestra en la Fig. 7, se identifica el equivalente de las tecnologías utilizadas en cada uno de los elementos de hardware integrados en la solución EVANI.

\section{RESULTADOS}

\subsection{Percepción}

Antes de entrar en los resultados es importante resaltar que, como apoyo al análisis de la propuesta frente al piloto, se realizó previamente una encuesta a expertos y una entrevista a la subdirectora de Salud Nutricional del Ministerio de Salud y Protección Social de Colombia.

La encuesta se aplicó en línea y se contó con el aporte de 16 expertos en el tema de SAN y que han tenido contacto con la desnutrición en primera infancia en el país. Como puntos clave de esta encuesta, se encontró lo siguiente:

Referenciaron 2 sistemas que realizan monitoreo de casos de desnutrición; SISVAN: Sistema de Vigilancia Nutricional SISVAN D.C.; este sistema es de alcance distrital y separa 2 tipos de medida, uno para menores de 7 años y otro para mayores de esta edad. Toma, además, de los valores nutricionales, otros datos relacionados con diferentes enfermedades.

MANGO: Sistema de Monitoreo y Nutrición de la Gobernación de Cundinamarca. Este consta de 2 componentes: una aplicación clienteservidor desde hospitales públicos y PC privados (alcaldías, colegios, etc.), y otra que es una aplicación web (depende de internet) para el registro de datos.

Si bien estos dos sistemas actualmente operan en las entidades del Estado, ninguna está enfocada al trabajo en territorio y funcionan siempre en línea. Acá encontramos la primera propuesta de valor de EVANI que es su opción offline y que permite levantar una alerta vía SMS. De la población encuestada, el $68 \%$ son profesionales con más de 5 años de experiencia, y este mismo porcentaje ha trabajado en procesos de antropometría en comunidades (territorio). Del total, solo el $50 \%$ conoce algún mecanismo de alerta en caso de desnutrición, y solo el $37 \%$ conoce la posibilidad de georreferenciar estos casos. El $81 \%$ cree que el sistema puede favorecer sustancialmente la mejora de datos estadísticos en territorio. El $56 \%$ han estado en contacto con casos de mortalidad infantil derivada de la desnutrición y el total estuvo de acuerdo 
Herramienta informática para notificación comunitaria como insumo para la generación de alertas en seguridad alimentaria y nutricional

con que el mecanismo que propone EVANI podría contribuir a salvar vidas.

En la entrevista con MINSALUD, se presenta el concepto y mecanismo de la herramienta EVANI, esto con el fin de identificar bajo la visión de las responsabilidades de esa subdirección, si la app puede generar algún impacto, aporte y/o algún tipo de evolución en los procesos existentes. A lo que responde: "Este podría ser uno de los mecanismos para el líder comunitario o la persona que esté en territorio. Que tenga la información del niño y pueda ir haciendo seguimiento. Eso es algo que no tienen las EPS y no saben cómo hacerlo, porque argumentan dificultades. En cambio, con la app, jel celular todos lo tienen!"

Se destaca en este encuentro lo siguiente: que la app puede ser un medio de apoyo para las entidades encargadas de atender los casos de desnutrición infantil; que los datos podrían convertirse en oficiales, una vez pasen un proceso de validación; que evidentemente es un apoyo inmediato en territorio para generar alertas; también que las entidades de control pueden monitorear y presionar para hacer efectivas las atenciones; finalmente, que puede hacerse extensivo el mecanismo para otro tipo de alertas en el ámbito de SAN.

\section{2 ¡En acción!}

Para evaluar el sistema en un ambiente real, se estableció contacto con un colegio público del orden distrital y un jardín infantil de naturaleza privada, con el fin de realizar un tamizaje a menores de 5 años y registrar los datos en la aplicación para validar el comportamiento. El colegio está ubicado en la localidad de San Cristóbal, en la parte alta de la montaña al sur de la capital; mientras que el jardín infantil se encuentra al otro extremo de la ciudad, en la localidad de Suba, Fig. 8.

El proceso ejecutado con los 2 entes colaboradores inició con el contacto de las personas a cargo de los grupos donde están los niños menores de 5 años, presentando el proyecto y entregando un consentimiento informado que solicita la autorización de los padres o acudientes responsables de cada niño y niña. Estos consentimientos se hicieron llegar a los acudientes, y una vez recibidos, se revisaron las autorizaciones para proceder con el agendamiento de la jornada de tamizaje.

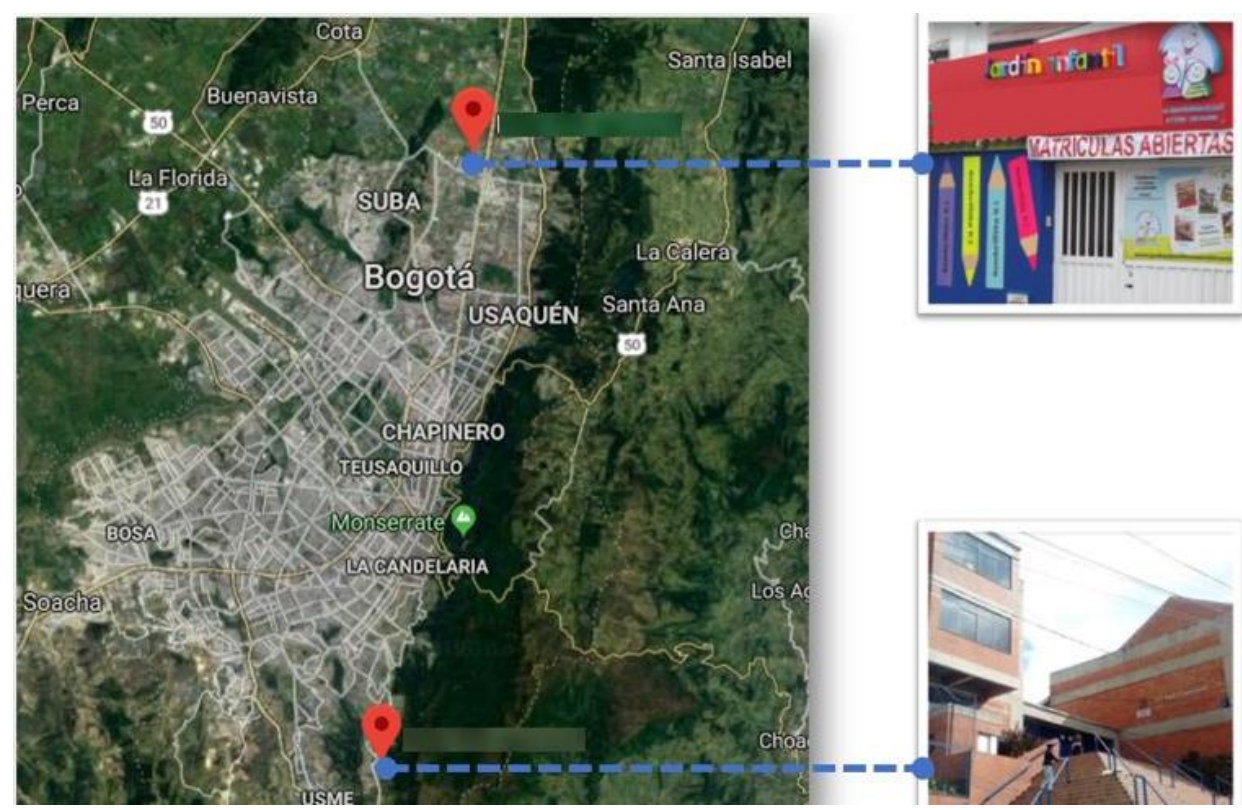

Fig. 8. Ubicación de las instituciones donde se ejecutó el piloto. Fuente: autor. 
Herramienta informática para notificación comunitaria como insumo para la generación de alertas en seguridad alimentaria y nutricional

Las jornadas de tamizaje se ejecutaron con 10 menores de 5 años en el colegio y 12 en el jardín. Para estos procesos se contó con básculas, tallímetros y cintas métricas. Estos instrumentos permitieron la captura de información de peso, talla, perímetro cefálico y perímetro braquial.

Una vez cargada la información y que la app realizara el diagnóstico, se obtuvieron los siguientes resultados:

En el indicador de talla para la edad, Fig. 9, se encuentra que el $91 \%$ de los participantes está en una talla adecuada para su edad, un caso se encuentra en riesgo de obtener baja talla, y otro ya está en baja talla para su edad.

En el indicador de peso para la edad, Fig. 10, se encuentra que el $95 \%$ de los participantes está en un peso adecuado para su edad, un caso está en riesgo de desnutrición global.

En el indicador de peso para la talla se encuentra que el $95 \%$ de los participantes está en un peso adecuado para su talla, sin embargo, un caso está en riesgo de desnutrición aguda y este, según la resolución de MINSALUD, es un caso de alerta, Fig. 11 y Fig. 12.

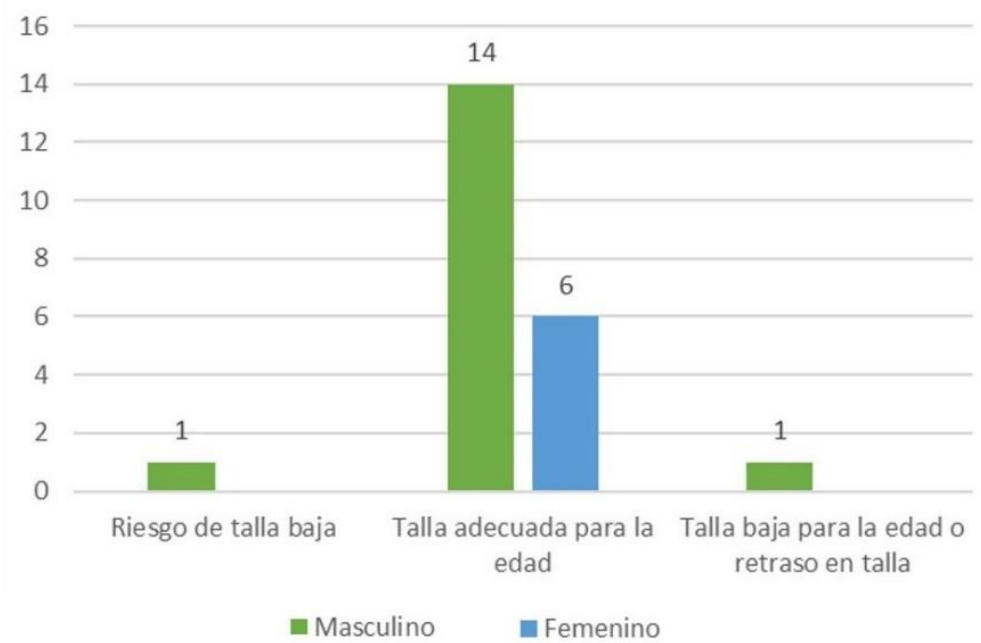

Fig. 9. Resultados del piloto para el indicador de talla para la edad por sexo. Fuente: autor.

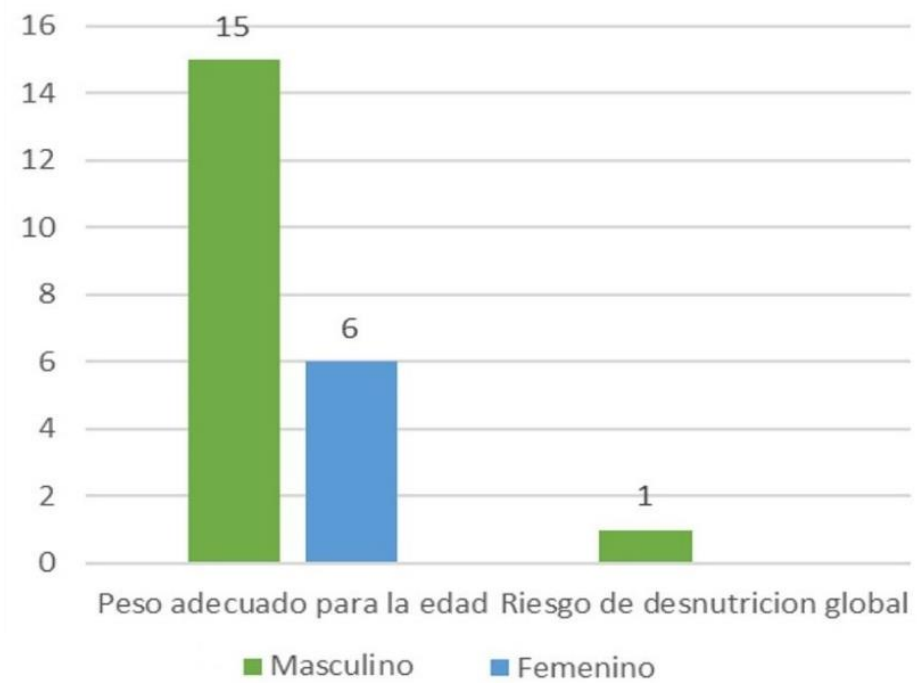

Fig. 10. Resultados del piloto para el indicador de peso para la edad por sexo. Fuente: autor. 
Herramienta informática para notificación comunitaria como insumo para la generación de alertas en seguridad alimentaria y nutricional

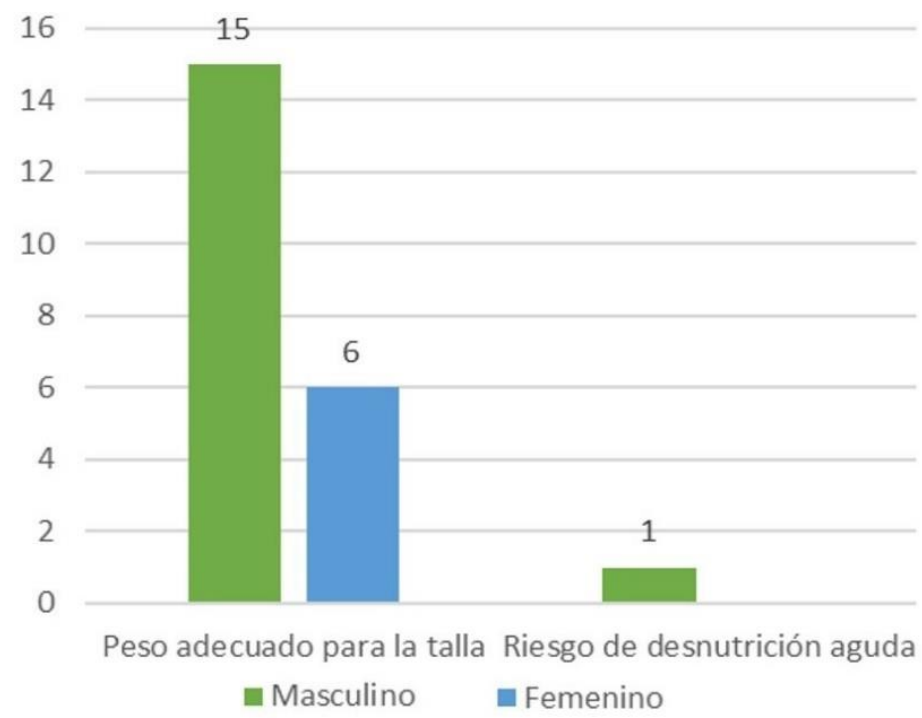

Fig. 11. Resultados del piloto para el indicador de peso para la talla por sexo. Fuente: autor.

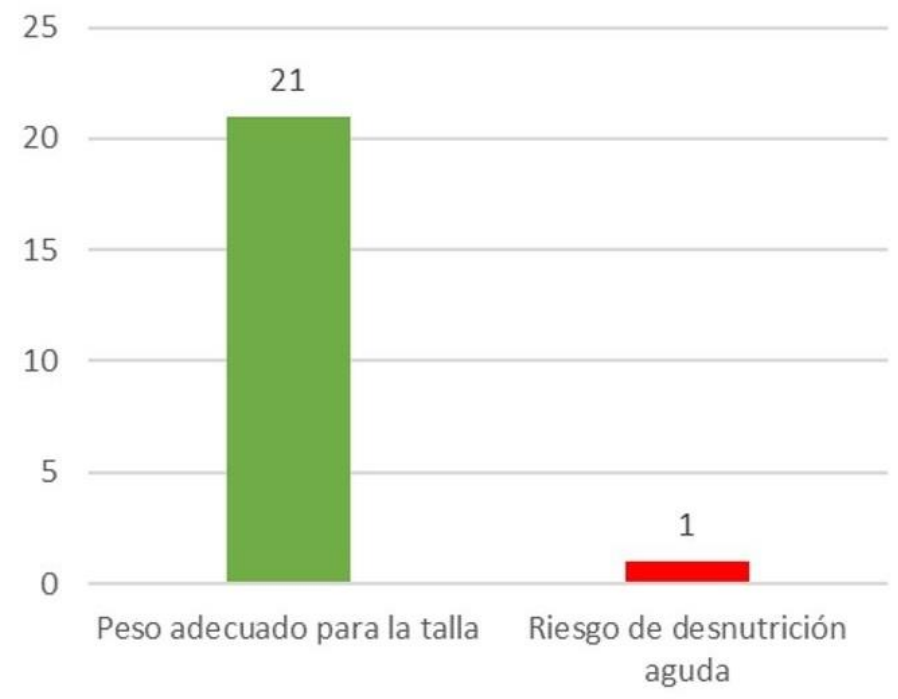

Peso adecuado para la talla $\mathbf{a}$ Riesgo de desnutrición aguda

Fig. 12. Resultados del piloto para el indicador de peso para la talla (PT) por prediagnóstico. Fuente: autor.

Al terminar los procesos de tamizaje y analizar los datos se encuentra un caso de alerta, y dentro del proceso propuesto, este caso se valida $y$ se generan las recomendaciones pertinentes al caso, inicialmente una consulta médica profesional para que evalúe y despliegue la ruta de atención que aplique según los procesos de los entes de salud.
Parte del compromiso con las instituciones y con los padres que aceptaron participar en las jornadas de tamizaje, era devolver una serie de recomendaciones según los resultados de cada niño o niña, por lo tanto, se entregó un informe con los resultados obtenidos y las recomendaciones del caso a cada institución. 
Herramienta informática para notificación comunitaria como insumo para la generación de alertas en seguridad alimentaria y nutricional

\section{DISCuSIóN}

Analizando lo desarrollado y los resultados obtenidos, se identifica claramente que EVANI puede apoyar el proceso de reporte de alertas tempranas por medio de comunidades en los territorios, las cuales serían validadas, divulgadas, e incorporadas a un seguimiento inicialmente por el monitor asignado a la zona y posteriormente a un ente de control interesado.

Sin embargo, es importante considerar que la población objetivo de esta fase es reducida frente al reto que se tiene a nivel nacional. Para esto, es importante que las entidades que decidan adoptar este sistema realicen nuevas jornadas para validar el comportamiento y escalar más asertivamente los requerimientos técnicos y tecnológicos que la puesta en marcha necesitaría.

Al realizar ejercicios de apropiación en comunidades, como es la propuesta de valor de EVANI, se generan procesos que llevan implícitamente innovación social y un uso de las TIC como aporte a los territorios. No obstante, el acompañamiento técnico y temático fue fundamental para identificar las necesidades de información, para llevar a cabo la implementación. Es muy importante tener presente las condiciones sociodemográficas de cada territorio, creencias étnicas y/o religiosas que pueden generar variaciones en los ejercicios de tamizaje.

Es importante resaltar que durante el proceso de construcción de EVANI se pudo identificar un caso de alerta real, reforzando la intensión del piloto $y$ validando que sí se presenta un impacto frente a otros mecanismos de reporte de casos. Ahora bien, para que el mecanismo funcione, no solo se debe reportar la alerta, sino que se debe garantizar la veracidad en la validación, la oportunidad en la atención $y$ el constante monitoreo de cada ente encargado, de lo contrario, no generaría un aporte más allá que la captura de datos.

Resultado de la carga de datos de muestra y las validaciones de funcionamiento de la aplicación, se encontraron oportunidades de mejora para una siguiente versión. Dentro de las posibilidades están nuevas funciones del sistema, como un reporte gráfico histórico de análisis con los seguimientos de los casos. También se identifica que se puede mejorar la desagregación de los datos capturados por etnia, personas con discapacidad, entre otros.

Esto aportaría al análisis de casos puntuales y a mejorar las estadísticas existentes, dejando un mensaje incluyente más explícito y permitiendo incorporar en el sistema un enfoque basado en derechos humanos, en donde se visibilicen poblaciones tradicionalmente excluidas [24].

Otra oportunidad consiste en implementar la solución en un esquema multiplataforma, que permita trabajar con dispositivos móviles de los sistemas operativos más comunes y estaciones de escritorio, para facilitar las tareas recurrentes propios del monitoreo y gestión de notificaciones. Así mismo, se podría sumar a las funcionalidades del sistema una batería de reportes adicionales, a partir de los indicadores capturados, ofreciendo así insumos que puedan ganar fuerza en las posibles tomas de decisiones de política pública en las regiones.

Se logró realizar una identificación y buen direccionamiento tecnológico para poder implementar la aplicación, de tal manera que cumpliera con los requerimientos funcionales planteados, $\mathrm{y}$ se abre así la posibilidad de que otros interesados puedan poner en práctica lo descrito en este documento para crear o fortalecer procesos de alertas tempranas y/o acceso a herramientas informáticas en territorio. 
Herramienta informática para notificación comunitaria como insumo para la generación de alertas en seguridad alimentaria y nutricional

\section{CONCLUSIONES}

Como resultado de las jornadas, se pudo identificar un caso en alerta por riesgo de desnutrición aguda, que es una de las condiciones que puede llevar a la muerte. Una identificación temprana del riesgo permite tomar acciones rápidas para poder mitigar la problemática. Esto hace parte de la propuesta de valor de EVANI.

El sistema EVANI app, para poder tener una operación óptima requiere del apoyo de algún ente de control dispuesto a realizar los seguimientos de los casos que se vayan a reportar, una entidad de orden público, grupo comunitario $\mathrm{y} / \mathrm{u}$ organización que desee hacer una tarea constante en la lucha contra la mortalidad infantil derivada de la desnutrición $y$, finalmente, acuerdos con las instituciones de salud y/o ICBF para poder garantizar una atención oportuna en los diferentes casos encontrados.

La aplicación es una apuesta en la innovación social, al poner al alcance de las zonas apartadas la posibilidad de levantar su voz ante una necesidad, apoyándose en la tecnología y las redes comunitarias.

Si bien EVANI es una solución con mirada social, requiere un mínimo de recursos para poder operar óptimamente, hacer sus mantenimientos y posteriores mejoras.

La app puede generar un gran impacto en la toma de datos en territorio y sería un buen referente o punto de partida para el manejo de alertas tempranas en SAN. Así se podrían plantear alianzas con entidades de diferentes sectores para captar información de seguimiento y acciones en los demás ejes temáticos. Es aquí donde se puede abrir opción de datos cada vez más precisos en la construcción de indicadores.

Existe una gran oportunidad de explotación de los datos con procesos de minería, con el fin de obtener comportamientos a través del tiempo, estudiar variables sociodemográficas, identificar factores políticos, entre otros que puedan ayudar a optimizar los procesos de promoción y prevención en la desnutrición infantil y, sobre todo, en la reducción de las tasas de mortalidad asociados.

\section{AGRADECIMIENTOS}

Este trabajo no se hubiese podido realizar sin la ayuda de varias personas de diferentes áreas del saber, principalmente debo mencionar la orientación técnica y de contexto que dio origen a la idea por parte de la nutricionista, conocedora de políticas públicas, Ángela Marcela Gordillo, de la Universidad Nacional de Colombia. En el área gráfica, aportaron sin algún interés distinto a llevar este piloto adelante, los diseñadores gráficos Mónica Vera Buitrago y Gabriel Clavijo Serrano. Igualmente, se hace extensiva la gratitud a Elisa María Cadena, que a pesar de sus múltiples compromisos dedicó un espacio para conocer y aportar con sus opiniones sobre EVANI.

\section{REFERENCIAS}

[1] R. Bisang and M. Campi, "Hambre, alta tecnología y desigualdad social: Un desafío a inicios del siglo XXI," Rev. Iberoam. Ciencia, Tecnol. y Soc. -CTS, vol. 5, no. 14, pp. 1-21, 2010.

[2] Organización de las Naciones Unidas para la Alimentación y la Agricultura, Fondo de las Naciones Unidas para la Infancia, Fondo Internacional de Desarrollo Agrícola, Programa Mundial de Alimentos, and Organización Mundial de la Salud, El estado de la seguridad alimentaria y la nutrición en el mundo. Roma: Organización de las Naciones Unidas para la Alimentación y la Agricultura, 2018.

[3] DANE, "Boletín técnico. Indicadores básicos de tenencia y uso de tecnologías de la información y comunicación en hogares y personas de 5 y más años de edad," Bogotá, 2018.

[4] E. F. Ruiz, Á. Proaño, O. J. Ponce, and W. H. Curioso, "Tecnologías móviles para la salud 
Herramienta informática para notificación comunitaria como insumo para la generación de alertas en seguridad alimentaria y nutricional

pública en el Perú: lecciones aprendidas," Rev. Peru. Med. Exp. Salud Publica, vol. 32, no. 2, pp. 264-272, 2015.

[5] Consejo Nacional de Política Económica y Social, República de Colombia, and Departamento Nacional de Planeación, "Política Nacional de Seguridad Alimentaria y Nutricional (PSAN)," Bogotá, 2007.

[6] Ministerio de Justicia y del Derecho, Decreto 1115. Colombia, 2014, p. 12.

[7] Gobierno de Colombia, "Plan Nacional de Seguridad alimentaria y nutricional (PNSAN) 2012-2019," 2012.

[8] Ministerio de Salud y Protección Social and Organización de las Naciones Unidas para la Alimentación y la Agricultura, "Documento metodológico para la construcción del subsistema de información de alertas en la seguridad alimentaria y nutricional,” Bogotá, 2013.

[9] Ministerio de Salud y Protección Social, Organización de las Naciones Unidas para la Alimentación y la Agricultura, and Observatorio de Seguridad Alimentaria y Nutricional, "Informe técnico del susbsistema de información de alertas en seguridad alimentaria y nutricional," 2014.

[10] J. C. Morales González and FoodFirst Information and Action Network, $L a$ exigibilidad del derecho humano a la alimentación. Qué es y cómo hacerla? Bogotá: FoodFirst Information and Action Network, 2015.

[11] Equipo de ISI Argonauta, A. F. Juan, C. B. Segade, P. G. Varela, and R. H. Lorite, "Guía para la incorporación del enfoque basado en derechos humanos en las intervenciones de cooperación para el desarrollo," Madrid, 2010.

[12] F. Mönckeberg B., "Desnutrición infantil y daño del capital humano," Rev. Chil. Nutr., vol. 41, no. 2, pp. 173-180, Jun. 2014. https://doi.org/10.4067/S0717-

75182014000200008 .

[13] Unión Internacional de Telecomunicaciones, "Question 14-2/2 - Mobile Health Solutions for Developing Countries," Geneva, Switzerland, 2010.

[14] E. J. O. Roca, "Aportes a la construcción participativa del Sistema de Información de Seguridad Alimentaria y Nutricional, Como base de la Política Publica de SAN en la isla de San Andrés islas, Caribe Colombiano," Universidad Nacional de Colombia, 2014.

[15] Food and Agriculture Organization of the United Nations Staff, Manual para el diseño $e$ implementación de un sistema de información para la seguridad alimentaria y alerta temprana-SISAAT. Food \& Agriculture Organization of the United
Nations, 2001.

[16] Acción contra el Hambre, "Guía para la Implementación de Sitios Centinela en Centroamérica. Un Sistema de Vigilancia y Alerta Temprana de la Inseguridad Alimentaria y nutricional basado en la comunidad," Guatemala, 2013.

[17] World Food Programme, "Alerta Temprana en Seguridad Alimentaria y Nutricional Luchando contra el hambre en el mundoIdentificación de variables y puntos críticos en El Salvador, Guatemala, Honduras y Nicaragua," World Food Programme, Inglaterra, 2017.

[18] S. Bravo Lasprilla, M. A. Valero Duboy, I. Pau de la Cruz, J. Duarte de Villa, M. Carrillo, and J. R. Blandino, "Sistema de información y comunicación para salud y nutrición de niños desatendidos en áreas rurales de Nicaragua," Inf. Technol. Int. Dev., vol. 8, no. 4, pp. 223-241, 2012.

[19] Observatorio del Caribe and Ecopetrol, "Alertas tempranas y prevención del riesgo: una tendencia mundial y un desafío para fomentar el desarrollo rural y la seguridad alimentaria de la región," Cartagena, 2013.

[20] Y. W. Galindo Galindo, "Desarrollo e Implementación del aplicativo movil APPEA basado en software libre para reducir los diagnósticos nutricionales del programa nacional Cuna Mas," Universidad Nacional José María Arguedas, 2014.

[21] C. Etienne, "La eSalud: aprovechar la tecnología en el camino hacia la cobertura universal de salud," Revista Panamericana de Salud Pública, vol. 35, no. 5, 6. pp. 317319, 2014.

[22] Ministerio de Salud y Protección Social, "Encuesta Nacional de Situación Nutricional," 2015. .

[23] Consejo Nacional de Política Económica y Social, República de Colombia, and Departamento Nacional de Planeación, "Estrategia para la implementación de los ODS en Colombia,” Bogotá, 2018.

[24] A. L. Álvarez Alvear, "La participación comunitaria en la formulación del Plan Decenal de Soberanía y Seguridad alimentaria y nutricional Nariño 2010-2019. Estudio de caso," Universidad Nacional de Colombia, 2016.

[25] M. C. Gasca Mantilla, L. L. Camargo Ariza, and B. Medina Delgado, "Metodología para el desarrollo de aplicaciones móviles," Rev. Tecnura, vol. 18, no. 40, p. 20, May 2014. https://doi.org/10.14483/udistrital.jour.tecnur a.2014.2.a02.

[26] R. M. U. Peñuela, "Las aplicaciones móviles utilizadas en nutrición," Perspect. en Nutr. humana, vol. 18, no. 2, p. 4, 2016. 
Herramienta informática para notificación comunitaria como insumo para la generación de alertas en seguridad alimentaria y nutricional

[27] X. Valente Adarme and C. Mercado Giménez, "El enfoque de los derechos humanos en las políticas públicas: ideas para un debate en ciernes," Cuad. CENDES, vol. 27, no. 74, pp. 51-79, 2010.

[28] Ministerio de Salud y Protección Social, Resolución 2465. Bogotá, Colombia, 2016, p. 47.
[29] P. Jahoda, "MPAndroidChart." GitHub, Austria, 2018. http://androidplot.com/

[30] AndroidPlot, "AndroidPlot." Androidplot, 2018. http://androidplot.com/

[31] Linus Torvalds, "Node.Js." Linux, 2018. https://nodejs.org/es/about/

[32] L. Torvalds, "Git." Git, 2019. https:/gitscm.com/downloads 